\title{
Dependence of Structure of Polyelectrolyte/Micelle Complexes upon Polyelectrolyte Chain Length and Micelle Size
}

\author{
Yingjie Lì and Paul L. Dubin* \\ Department of Chemistry, Indiana University-Purdue University, \\ Indianapolis, Indiana 46202 \\ Herbert Dautzenberg, Ursula Luck, and Jurgen Hartmann \\ Max Planck Institute for Colloid and Interface Research, Teltow-Seehof 1530, Germany \\ Zdenek Tuzar \\ Institute of Macromolecular Chemistry, Prague, Czech Republic \\ Received March 28, 1995; Revised Manuscript Received July 24, 1995 ${ }^{\otimes}$
}

\begin{abstract}
Static light scattering and dynamic light scattering were used to investigate the effect of polyelectrolyte chain length and micelle size on the complex formation between a strong polyelectrolyte, poly (dimethyldiallylammonium chloride) (PDMDAAC), and oppositely charged mixed micelles of Triton X-100 (TX100) and sodium dodecyl sulfate (SDS). Although an increase in the molecular weight of PDMDAAC led to high molar mass of the complex, the degree of binding, defined as the mass ratio of micelle to PDMDAAC in the complex, decreased with increasing PDMDAAC molecular weight. The relation between the molar mass and hydrodynamic size indicated that the complex was compact. With increasing micelle size, the structure of the complex changed from one in which a PDMDAAC molecule binds several micelles to one in which large micelles adsorb one or more low molecular weight PDMDAAC molecule(s). Complexes formed from high molecular weight PDMDAAC fractions and large micelles showed a tendency for coacervation.
\end{abstract}

\section{Introduction}

Complex formation between polyelectrolytes and oppositely charged surfactants has been a subject of intense research effort. ${ }^{1-6}$ Most of the early studies involved surfactants well below their critical micelle concentration $(\mathrm{cmc})$. This is because the strong electrostatic interaction between polyelectrolyte and surfactant results in precipitation at surfactant concentrations even well below the cmc. The early precipitation prevents a detailed investigation and hinders the application of many techniques well established in colloid science. Such strong electrostatic interaction may be attenuated by using mixed micelles of nonionic and ionic surfactants. ${ }^{7}$ For example, poly (dimethyldiallylammonium chloride) (PDMDAAC) and mixed micelles of sodium dodecyl sulfate (SDS) and Triton X-100 (TX100) can form soluble complexes under a wide range of conditions. A number of techniques have been used to study this system. ${ }^{8-17}$

It is not a priori clear how the relative sizes of polyelectrolyte and micelle affect the structure of the complex. Polymer/micelle complexes were described by Cabane and Duplessix as a "necklace of beads". ${ }^{18}$ However, this model must break down if the size of the micelles becomes similar to that of the polymer, resulting from either a decrease in the length of polymer or an increase in the micelle size. The terminology itself depends on scale: the term "binding" prevails in the literature on polymer/micelle complex formation (large polymers and small colloids), while "adsorption" is more familiar in the context of the interaction of a polymer with a flat surface (large colloids). It is interesting to

\footnotetext{
* To whom correspondence should be addressed.

† Current address: Century International Adhesives and Coatings Corp., 802 Harmon Ave., Columbus, OH 43223.

$\otimes$ Abstract published in Advance ACS Abstracts, September 1, 1995.
}

know whether there is some ratio of polymer to micelle size at which one conceptual approach becomes more appropriate than the other. PDMDAAC/TX100-SDS seems to provide a system with which such questions may be addressed. We have prepared PDMDAAC fractions of varying molecular weight (MW), ${ }^{19}$ and the size of TX100-SDS mixed micelles can be controlled by ionic strength, ${ }^{20}$ although the micelle composition must be changed concomitantly in order to keep the system in the region of soluble complexes. In a previous paper, we ${ }^{16}$ reported the observation of a transition from intrapolymer complex to interpolymer complex with increasing polymer concentration and found a strong dependence of this transition on polyelectrolyte molecular weight. Specifically, the polyelectrolyte concentration at which the transition occurs decreases with increasing polyelectrolyte molecular weight. In this paper we study the structure of the complex as a function of PDMDAAC chain length and micelle size. The study was performed at low polymer concentration so that no interpolymer complex could form.

\section{Experimental Section}

Materials and Solution Preparation. A commercial sample of PDMDAAC, Merquat 100 (Calgon Corp., Pittsburgh, PA), with a nominal molecular weight of $200 \mathrm{~K}$ and a polydispersity of $M_{\mathrm{w}} / M_{\mathrm{n}} \sim 10$ was fractionated via preparative GPC, and the fractions were characterized by static and dynamic light scattering, viscosity, GPC, and electrophoretic light scattering. ${ }^{19}$ Table 1 shows molecular characteristics of the PDMDAAC fractions used in this study. TX100 was purchased from Aldrich, and SDS from Fluka. Ionic strength was adjusted by using $\mathrm{NaCl}$ from Fisher. Mill-Q water was used throughout this work. TX100, SDS, and $\mathrm{NaCl}$ were used without further purification.

Solutions were prepared by adding $60 \mathrm{mM}(100 \mathrm{mM})$ SDS in $0.4 \mathrm{M}(1.0 \mathrm{M}) \mathrm{NaCl}$ to a solution of $40 \mathrm{mM}$ TX100 and 0.6 g/L PDMDAAC to bring the $Y$ value to $0.3(0.5)$, where $Y$ is defined as [SDS]/([SDS] + [TX100]). Such $Y$ values were chosen based on the turbidity measurements to be discussed 
Table 1. Description of the PDMDAAC Fractions and TX100-SDS Micelles Used in This Study

\begin{tabular}{lrcc}
\hline & $10^{-3} M_{\mathrm{w}}$ & $R_{\mathrm{g}}(\mathrm{nm})$ & $R_{\mathrm{h}}(\mathrm{nm})$ \\
\hline PDMDAAC, 0.4 M NaCl & \\
& 22 & $11^{d}$ & 4 \\
& 50 & $14^{d}$ & 6 \\
& 110 & $21^{d}$ & 8 \\
& 200 & $25^{d}$ & 12 \\
& 370 & $26^{d}$ & 14 \\
& 590 & $29^{d}$ & 18 \\
TX100-SDS, $Y=0.3,0.4 \mathrm{M} \mathrm{NaCl}^{c}$ & 1300 & $40^{d}$ & 27 \\
TX100-SDS, $Y=0.5,1.0 \mathrm{M} \mathrm{NaCl}^{c}$ & 1110 & 16 & 9 \\
& & 35 & 21
\end{tabular}

${ }^{a}$ Values from ref $19 .{ }^{b} R_{\mathrm{h}}$ from dynamic light scattering. ${ }^{c} \mathrm{Re}-$ sults from dynamic and static light scattering. $(\mathrm{d} n / \mathrm{d} c)$ of micelles is $0.137{ }^{d}$ Interpolated values based on the results in $0.50 \mathrm{M} \mathrm{NaCl}$ in ref 19 .

later. After at least $2 \mathrm{~h}$ of stirring, the solutions were diluted 10 times using $0.4 \mathrm{M}$ (or $1.0 \mathrm{M}$ ) NaCl, to yield final solutions with a polymer concentration $C_{\mathrm{p}}=0.0466 \mathrm{~g} / \mathrm{L}$ (or $0.0428 \mathrm{~g} / \mathrm{L}$ ) and $[T X 100]=3.1 \mathrm{mM}$ (or $2.8 \mathrm{mM}$ ). Since the cmc of TX100SDS mixed micelles is expected to be less than $0.2 \mathrm{mM}$, i.e., $\sim 15$ times smaller than the surfactant concentrations employed here, the system we studied is a polyelectrolyte/micelle system. The solution under such conditions is an excess micelle system, and intrapolymer complexes are predominant. ${ }^{16,17}$

Dynamic Light Scattering. The diluted solutions were passed through $0.45-\mu \mathrm{m}$ filters (Life Science Products). Most of the dynamic light scattering measurements were carried out at $24 \pm 1{ }^{\circ} \mathrm{C}$ and at angles from 45 to $120^{\circ}$ using a Brookhaven Instruments system equipped with a 72-channel digital correlator (BI-2030AT) and an Omnichrome air-cooled $200 \mathrm{~mW}$ argon ion laser operating at a wavelength in vacuum $\lambda_{0}=488 \mathrm{~nm}$.

In the self-beating mode of dynamic light scattering, the measured photoelectron count autocorrelation function $G^{(2)}$. $(\tau, q)$ for a detector with a finite effective photocathode area has the form ${ }^{21}$

$$
G^{(2)}(\tau, q)=N_{\mathrm{s}}\langle n\rangle^{2}\left(1+b\left|g^{(1)}(\tau, q)\right|^{2}\right)
$$

where $g^{(1)}(\tau, q)$ is the first-order scattered electric field $\left(E_{\mathrm{g}}\right)$ time correlation function; $\tau$, the delay time; $\langle n\rangle$, the mean counts per sample; $N_{\mathrm{s}}$, the total number of samples; $A\left(=N_{\mathrm{s}}\langle n\rangle^{2}\right)$, the base line; $b$, a spatial coherence factor depending upon the experimental setup and taken as an unknown parameter in the data fitting procedure; and $q=\left(4 \pi n / \lambda_{0}\right) \sin (\theta / 2)$, with $n$ and $\theta$ being the refractive index of the scattering medium and the scattering angle, respectively.

For a solution of polydisperse particles, $g^{(1)}(\tau, q)$ has the form ${ }^{21}$

$$
\left|g^{(1)}(\tau, q)\right|=\int_{0}^{\infty} G(\Gamma, q) \mathrm{e}^{-(\Gamma(q) \tau)} \mathrm{d} \Gamma
$$

where $G(\Gamma, q)$ is the normalized distribution of line width $\Gamma$ measured at a fixed value of $q$. In the present study, a CONTIN algorithm was used to obtain the average $\Gamma$ and its distribution of the complex mode and the free micelle model. ${ }^{22}$

The line width $\Gamma$ at a finite concentration $C$ and a finite value of $q$ is related to the translational diffusion coefficient at infinite dilution and $q \rightarrow 0$ extrapolation, $D_{0}$, by ${ }^{23}$

$$
\Gamma=D_{0} q^{2}\left(1+f R_{\mathrm{g}}^{2} q^{2}\right)\left(1+k_{\mathrm{d}} C\right)
$$

where $f$ is a dimensionless number which depends upon the chain structure, polydispersity, and solvent quality; $\boldsymbol{R}_{\mathrm{g}}$ is the radius of gyration; and $k_{\mathrm{d}}$ is an average system-specific second virial (diffusion) coefficient which combines the hydrodynamic and thermodynamic factors. Therefore, in order to get $D_{0}$, extrapolations of both $C \rightarrow 0$ and $q \rightarrow 0$ are required. The hydrodynamic radius, $R_{\mathrm{h}}$, can then be obtained by using Stokes-Einstein relation

$$
D_{0}=k_{\mathrm{B}} T /\left(6 \pi \eta R_{\mathrm{h}}\right)
$$

where $k_{\mathrm{B}}$ is the Boltzmann constant, $T$ is the absolute temperature, and $\eta$ is the solvent viscosity.

In the present study, only one concentration is used. Therefore, the $R_{\mathrm{h}}$ values presented in this paper are only apparent ones due to the lack of correction for particle interaction effects. However, the concentration of PDMDAAC in this study is fairly low (typically $0.05 \mathrm{~g} / \mathrm{L}$ ). Therefore the lack of concentration extrapolation should not introduce a significant error.

Static Light Scattering. A Sofica 42000 (Wippler and Scheibling, Strasbourg, France) with a $\mathrm{He}-\mathrm{Ne}$ laser of $5 \mathrm{~mW}$ was used for static light scattering. The solutions were passed through $0.65-\mu \mathrm{m}$ cellulose filters. Static light scattering measurements were performed at $22^{\circ}$ and at angles from 30 to $150^{\circ}$. The excess Rayleigh ratio $R$ at $q \rightarrow 0$ can be obtained from either $\log R$ vs $q^{2}$ (Guinier plot) or $1 / R$ vs $q^{2}$. The results from the two methods agreed with each other within $\pm 1 \%$.

If we assume that the concentration is low enough so that the concentration effect is negligible, we can treat the complex solution as a bimodal system of complex and free micelles with $0.4 \mathrm{M}(1.0 \mathrm{M}) \mathrm{NaCl}$ as the solvent. $R$ at $q \rightarrow 0$ has the form ${ }^{21}$

$$
R=K_{\mathrm{x}} \mathrm{c}_{x} M_{\mathrm{w}, \mathrm{x}}+K_{\mathrm{m}} c_{\mathrm{m}, \mathrm{f}} M_{\mathrm{w}, \mathrm{m}}
$$

where $K_{\mathrm{x}}$ and $K_{\mathrm{m}}$ are the contrast factors of complex and micelle, respectively; $c_{\mathrm{x}}$ and $c_{\mathrm{m}, \mathrm{f}}$ are the mass concentrations of complex and free micelle, respectively; $M_{\mathrm{w}, \mathrm{x}}$ and $M_{\mathrm{w}, \mathrm{m}}$ are the weight-average molar masses of complex and micelle, respectively; and $K_{\mathrm{x}}=4 \pi^{2} n^{2}(\mathrm{~d} n / \mathrm{d} c)_{\mathrm{x}}{ }^{2} /\left(N_{\mathrm{A}} \lambda_{0}^{4}\right)$ and $K_{\mathrm{m}}=4 \pi^{2} n^{2}$. $(\mathrm{d} n / \mathrm{d} c)_{\mathrm{m}}{ }^{2} /\left(N_{\mathrm{A}} \lambda_{0}{ }^{4}\right)$, with $(\mathrm{d} n / \mathrm{d} c)_{\mathrm{x}},(\mathrm{d} n / \mathrm{d} c)_{\mathrm{m}}$, and $N_{\mathrm{A}}$ being the refractive index increment of complex, the refractive index increment of micelle, and Avogadro's number, respectively. $(\mathrm{d} n /$ dc) $x_{x}$ can be approximated by

$$
(\mathrm{d} n / \mathrm{d} c)_{\mathrm{x}}=1 /(1+\gamma)(\mathrm{d} n / \mathrm{d} c)_{\mathrm{p}}+\gamma /(1+\gamma)(\mathrm{d} n / \mathrm{d} c)_{\mathrm{m}}
$$

where $\gamma$, called the degree of binding, is the mass ratio of surfactant to PDMDAAC in the complex, and $(\mathrm{d} n / \mathrm{d} c)_{\mathrm{p}}$ is the refractive index increment of PDMDAAC. Since the mass of the complex is usually predominantly micelles, ${ }^{15}$ i.e., $\gamma \gg 1$, $(\mathrm{d} n / \mathrm{d} c)_{\mathrm{x}} \approx(\mathrm{d} n / \mathrm{d} c)_{\mathrm{m}}$, which has a value of 0.137 .14 Previous work has shown that interpolymer complexes do not form under the conditions used in the present study. ${ }^{16}$ Therefore in the intrapolymer complex region, $M_{\mathrm{w}, \mathrm{x}}=M_{\mathrm{w}, \mathrm{p}}(1+\gamma)$ and $c_{\mathrm{x}}$ $=c_{\mathrm{p}}(1+\gamma)$, where $M_{\mathrm{w}, \mathrm{p}}$ is the molecular weight of PDMDAAC. Equation 5 can be written as

$$
\begin{aligned}
R /\left(K_{\mathrm{m}} c_{\mathrm{p}}\right) & =M_{\mathrm{w}, \mathrm{p}}(1+\gamma)^{2}+M_{\mathrm{w}, \mathrm{m}} c_{\mathrm{m}, \mathrm{f}} / c_{\mathrm{p}} \\
& =M_{\mathrm{w}, \mathrm{p}}(1+\gamma)^{2}+M_{\mathrm{w}, \mathrm{m}}\left(c_{\mathrm{m}} / c_{\mathrm{p}}-\gamma\right)
\end{aligned}
$$

where $c_{\mathrm{m}}$ is the total micelle concentration.

In eq 7 all quantities except for $\gamma$ are experimentally known. Therefore, $\gamma$ can be determined by solving eq 7 as

$$
\begin{array}{r}
\gamma=\left(M_{\mathrm{w}, \mathrm{p}} / M_{\mathrm{w}, \mathrm{p}}-2\right) / 2+\left[\left(M_{\mathrm{w}, \mathrm{m}} / M_{\mathrm{w}, \mathrm{p}}-2\right)^{2} / 4+\right. \\
\left.R /\left(K_{\mathrm{m}} c_{\mathrm{p}}\right) / M_{\mathrm{w}, \mathrm{p}}-\left(M_{\mathrm{w}, \mathrm{m}} / M_{\mathrm{w}, \mathrm{p}}\right)\left(c_{\mathrm{m}} / c_{\mathrm{p}}\right)-1\right]^{1 / 2}
\end{array}
$$

and the value of $\gamma$ provides $M_{\mathrm{w}, \mathrm{x}}$ as $M_{\mathrm{w}, \mathrm{x}}=M_{\mathrm{w}, \mathrm{p}}(1+\gamma)$.

\section{Results and Discussion}

Figure 1 shows the turbidity of PDMDAAC/TX100SDS (reported as $100-\% T$ ) with increasing $Y$. In 0.40 $\mathrm{M} \mathrm{NaCl}$, no interaction is observed until $Y$ reaches 0.23 , denoted as $Y_{c}$. Soluble complexes are observed when $Y$ is between 0.23 and $c a .0 .4$. At $Y>Y_{p}=0.4$ phase separation takes place as indicated by a sharp turbidity increase. In $1.00 \mathrm{M} \mathrm{NaCl}$, both $Y_{\mathrm{c}}$ and $Y_{\mathrm{p}}$ are shifted to higher values. In order to investigate the soluble complexes, $Y=0.3$ and 0.5 were chosen for $0.40 \mathrm{M} \mathrm{NaCl}$ and $1.0 \mathrm{M} \mathrm{NaCl}$ solutions, respectively. 


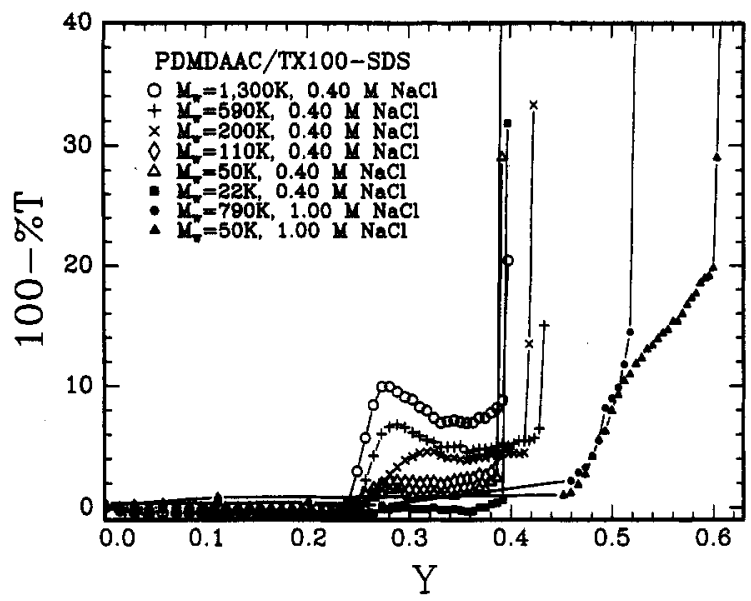

Figure 1. Turbidity of PDMDAAC/TX100-SDS as a function of $Y$ in 0.40 and $1.00 \mathrm{M} \mathrm{NaCl}$, respectively.
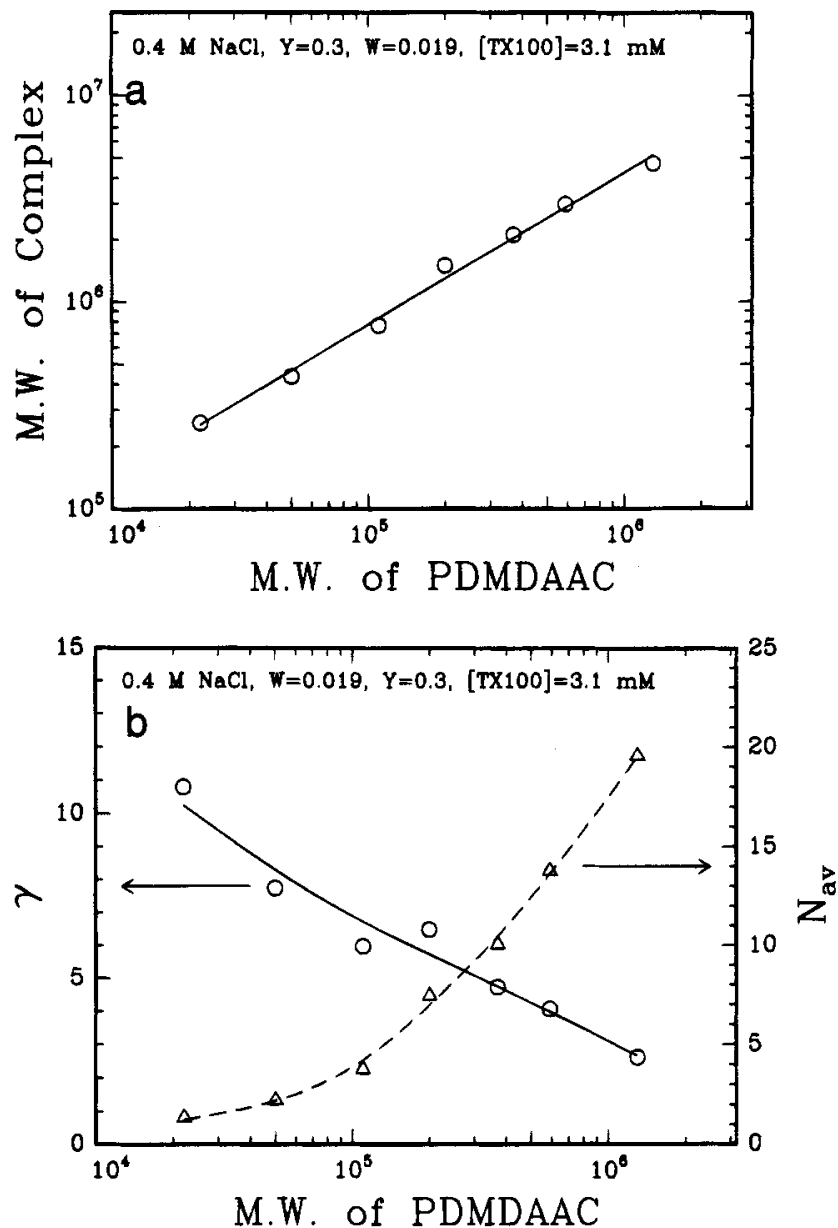

Figure 2. (a) Molar mass of the complex; (b) degree of binding, $\gamma$ (defined as the mass ratio of bound micelle to PDMDAAC in a complex) and average number of bound micelles in a complex, $N_{\text {av }}$, both as a function of the molecular weight of PDMDAAC fractions in $0.4 \mathrm{M} \mathrm{NaCl}$. $W$ is defined as the bulk weight ratio of PDMDAAC to TX100-SDS.

Figure 2a shows the molar mass of complexes as a function of PDMDAAC molecular weight. The results can be fitted to $M_{\mathrm{w}, \mathrm{x}} \sim M_{\mathrm{w}, \mathrm{p}}{ }^{0.74}$. If we assume that the bound and the free micelles have the same aggregation number (the absence of appreciable structural changes of TX100-SDS micelles upon binding to PDMDAAC is qualitatively supported by preliminary cryo-TEM stud${ }^{2} \mathrm{~s}^{24}$ ), the average number of bound micelles in a complex $\left(N_{\mathrm{av}}\right)$ can be calculated. The results are shown in Figure 2b. The fraction with $M_{\mathrm{w}}=22 \mathrm{~K}$ binds on average less than 2 micelles. As shown in Table $1, R_{\mathrm{h}}$ for this fraction is only $4 \mathrm{~nm}$, even smaller than the micelle size $(9 \mathrm{~nm})$. Therefore, "adsorption of PDMDAAC to the micelle surface" might be a more appropriate term than "binding of micelle to polymer". Although $N_{\text {av }}$ increases with PDMDAAC molecular weight, the degree of binding $(\gamma)$ decreases. If PDMDAAC chains within the complex adopted a highly extended (rodlike) conformation, we would expect $M_{\mathrm{w}, \mathrm{x}}$ to be a linear function of $M_{\mathrm{w}, \mathrm{p}}$, i.e., $\gamma$ independent of $M_{\mathrm{w}, \mathrm{p}}$. The decrease in $\gamma$ with increasing $M_{\mathrm{w}, \mathrm{p}}$ implies that the PDMDAAC chain in the complex is not highly elongated. One might propose that the PDMDAAC chains tend to maintain a random coil conformation in order to minimize configuration entropy loss during complex formation. Micelles that reside near the center of mass of a PDMDAAC chain must perturb the chain conformation, while a more favorable case might be for some bound micelles to reside at the outer boundary of the PDMDAAC domain. Although high molecular weight PDMDAAC molecules can bind more micelles far from the center of mass, this distal binding capacity divided by the number of PDMDAAC repeating units is larger for low molecular weight PDMDAAC fractions, leading to a larger degree of binding $\gamma$.

We may estimate the net charge of the complex from $\gamma$. In order to completely neutralize the positive charge in PDMDAAC, $\gamma$ should be 11.2, which is greater than the $\gamma$ values obtained here. Therefore, we would expect a complex with positive charge. In contrast, previous studies ${ }^{17,25}$ with electrophoretic light scattering indicating that the complex had a net negative charge when $W$ is less than 0.09 in $0.4 \mathrm{M} \mathrm{NaCl}$ with $Y=0.3$, where $W$ is defined as the weight ratio of PDMDAAC to TX100-SDS. This discrepancy can be reconciled if we consider the distribution of SDS in TX100-SDS micelles. Micelles with more SDS than the average value (" $y$ " $>Y_{\mathrm{av}}$ ) may have a higher binding constant and so preferentially complex with PDMDAAC. If $Y>0.3$, the net charge for a given $\gamma$ will be more negative. Similar speculations about micelle compositional polydispersity were made earlier by Dubin et al.7,15

Our previous study ${ }^{16}$ has shown a typical bimodal distribution from CONTIN analysis of the dynamic light scattering results of the PDMDAAC/TX100-SDS system with $Y=0.30$ and $0.40 \mathrm{M} \mathrm{NaCl}$ (see Figure 6 of ref 16). From the distribution, $R_{\mathrm{h}}$ of the complex and the free micelles can be determined. The $R_{\mathrm{h}}$ of value of $c a$. $9 \mathrm{~nm}$ for free micelles is in agreement with previous results. ${ }^{13}$ The size of the complex increases with increasing PDMDAAC molecular weight, while the size of the free TX100-SDS micelles remains essentially unchanged (see Figure 2 of ref 16). Figure 3 shows the relation between $R_{\mathrm{h}}$ and the molar mass of the complex. For a macromolecular solution, a scaling relation $R_{\mathrm{h}} \sim$ $M_{\mathrm{w}}{ }^{v}$ holds, with $v$ depending upon the macromolecular conformation. $v$ has values of $0.6,0.5$, and 0.33 for a coil in a good solvent, an unperturbed Gaussian coil, and a uniform sphere, respectively. ${ }^{26}$ The straight line in Figure 3 yields $R_{\mathrm{h}} \sim M_{\mathrm{w}, \mathrm{x}}{ }^{0.44}$. Therefore, $v$ is between the values for unperturbed Gaussian coils and uniform spheres, indicating that the complex is rather compact.

As shown in Table 1 , the micelle in $1.0 \mathrm{M} \mathrm{NaCl}$ has a size of $R_{\mathrm{h}}=21 \mathrm{~nm}$ and a molar mass of $1110 \mathrm{~K}$, both of which are larger than the corresponding values for most of the PDMDAAC fractions used here. At this high ionic strength the electrostatic interactions between PDMDAAC and TX100-SDS are reduced; to reach 


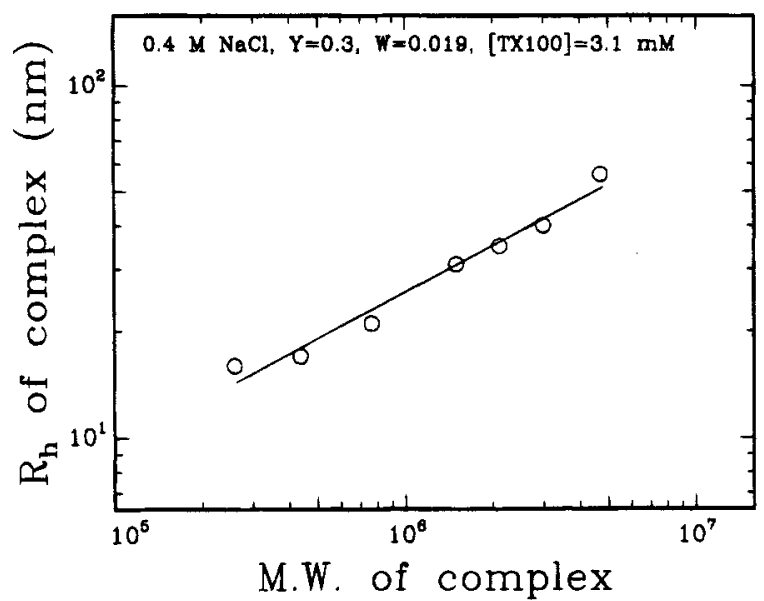

Figure 3. Dependence of $R_{\mathrm{h}}$ upon molar mass of the complex in $0.4 \mathrm{M} \mathrm{NaCl}$.

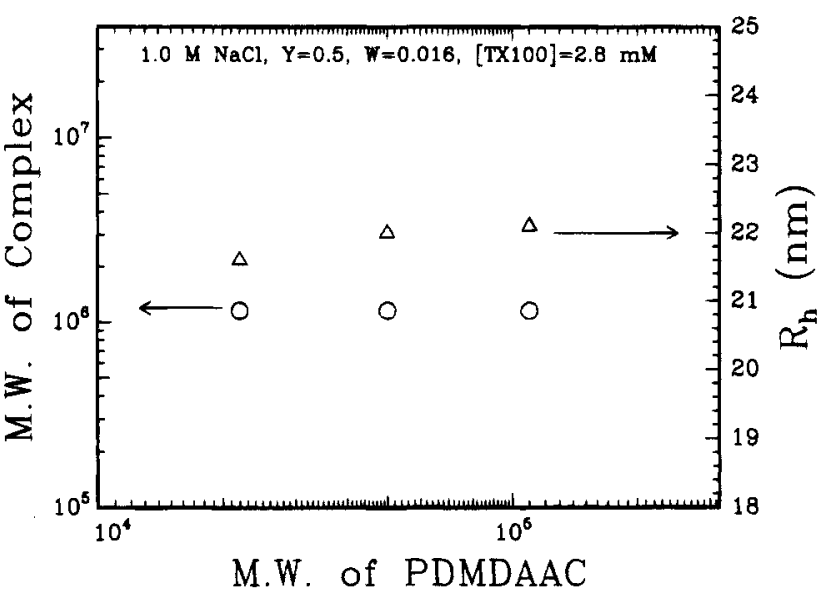

Figure 4. Molar mass of the complex and $R_{\mathrm{h}}$ of the system as a function of PDMDAAC molecular weight in $1.0 \mathrm{M} \mathrm{NaCl}$.

complexation, this must be offset by increasing $Y(0.5$ in $1.0 \mathrm{M} \mathrm{NaCl}$ ). Complexes in $1.0 \mathrm{M} \mathrm{NaCl}$ showed very different solubility behavior from those at $I=0.40 \mathrm{M}$. Upon dilution with $1.0 \mathrm{M} \mathrm{NaCl}$, soluble complex solutions prepared from PDMDAAC with molecular weight $\geq 200 \mathrm{~K}$ coacervated. Although we do not have a conclusive explanation for this observation, most coacervation theories predict that increased molecular weight of the components facilitates coacervation. ${ }^{27-29}$ Consequently, light scattering measurements were only performed on solutions with PDMDAAC molecular weight less than $200 \mathrm{~K}$.

Figure 4 shows that neither the molar mass nor $R_{\mathrm{h}}$ of the complexes formed from the three low molecular weight PDMDAAC fractions in $1.0 \mathrm{M} \mathrm{NaCl}$ shows significant difference from free micelles (since the size of the complex is close to that of the micelle, $R_{\mathrm{h}}$ presented in Figure 4 is an average of free micelles and adsorbed micelles). This result can be understood as adsorption of small PDMDAAC molecules to a single large micelle. Since the bridging of two micelles by a PDMDAAC molecule would lead to a complex with a molar mass of at least $2000 \mathrm{~K}$ in $1.0 \mathrm{M} \mathrm{NaCl}$, Figure 4 suggests that there is only one micelle in each complex. However, we could not rule out the possibility of more than one PDMDAAC molecule adsorbed on one micelle. This is particularly so if we realize that the bulk number ratio of PDMDAAC molecules to micelles in the solutions has values of $0.80,0.35$, and 0.16 for the three PDMDAAC fractions with $M_{\mathrm{w}, \mathrm{p}}=22 \mathrm{~K}, 50 \mathrm{~K}$, and $110 \mathrm{~K}$, respectively.

\section{Concluding Remarks}

Static light scattering and dynamic light scattering were used to determine the molar mass and hydrodynamic size of complexes formed from PDMDAAC and TX100-SDS mixed micelles. Although high molecular weight PDMDAAC fractions resulted in complexes of high molar mass, the degree of surfactant binding decreased with increasing PDMDAAC molecular weight. The relation between the molar mass and $R_{\mathrm{h}}$ indicated that the complex was compact. With increasing micelle size, the system changed from one in which a single PDMDAAC molecule binds more than one micelle to one in which large micelles adsorb one (or possibly more) low molecular weight PDMDAAC molecule(s).

Acknowledgment. The support of Grant DMR9311433 from the National Science Foundation (Divisions of Materials Research and Chemical Transport Systems) is gratefully acknowledged.

\section{References and Notes}

(1) Robb, I. D. In Anionic Surfactants, Physical Chemistry of Surfactant Action; Lucassen-Reynders, E. H., Ed.; Marcel Dekker: New York, 1981; p 109

(2) Goddard, E. D. Colloids Surf. 1986, 19, 301

(3) Hayakawa, K.; Kwak, J. C. T. In Cationic Surfactants. Physical Chemistry; Rubingh, D. N., Holland, P. M., Eds.; Marcel Dekker: New York, 1991; Chapter 5, p 189.

(4) Piculell, B.; Lindman, B. Adv. Colloid Interface Sci. 1992, 41, 149.

(5) Lindman, B.; Thalberg, K. In Interactions of Surfactants with Polymers and Proteins; Goddard, E. D., Ananthapadmanabhan, K. P., Eds.; CRC Press: Boca Raton, FL, 1993; Chapter 5 .

(6) Li, Y.; Dubin, P. L. In Structure and Flow in Surfactant Solutions; Herb, C. A., Prud'homme, R. K., Eds.; ACS Symposium Series 578; American Chemical Society: Washington, DC, 1994; Chapter 23, p 320.

(7) Dubin, P. L.; Oteri, R. J. Colloid Interface Sci. 1983, 95, 453.

(8) Dubin, P. L.; Davis, D. D. Macromolecules 1984, 17, 1294.

(9) Dubin, P. L.; Davis, D. D. Colloids Surf. 1985, 13, 113.

(10) Dubin, P. L.; Rigsbee, D. R.; McQuigg, D. W. J. Colloid Interface Sci. 1985, 105, 509.

(11) Dubin, P. L.; Rigsbee, D. R.; Gan, L.-M.; Fallon, M. A Macromolecules 1988, 21, 2555.

(12) Dubin, P. L.; Thé, S. S.; McQuigg, D. W.; Chew, C. H.; Gan, L.-M. Langmuir $1989,5,89$.

(13) Dubin, P. L.; Vea, M. E.; Fallon, M. A.; Thé, S. S.; Rigsbee, D. R.; Gan, L.-M. Langmuir 1990, 6, 1422

(14) Dubin, P. L.; Thé, S. S.; McQuigg, D. W.; Gan, L.-M.; Chew, C. H. Macromolecules 1990,23, 2500 .

(15) Xia, J.; Zhang, H.; Rigsbee, D. R.; Dubin, P. L.; Shaikh, T. Macromolecules 1993, 26, 2759.

(16) Li, Y.; Xia, J.; Dubin, P. L. Macromolecules 1994, 27, 7049

(17) Li, Y.; Dubin, P. L.; Havel, H. A.; Edwards, S. L.; Dautzenberg, H. Macromolecules 1995, 28, 3098.

(18) Cabane, B.; Duplessix, R. J. Phys. (Fr.) 1982, 43, 1529

(19) Xia, J.; Dubin, P. L.; Edwards, S.; Havel, H. A. J. Polym. Sci. Polym. Phys. Ed. 1995, 33, 1117.

(20) Dubin, P. L.; Principi, J. M.; Smith, B. A.; Fallon, M. A. J. Colloid Interface Sci. 1989, 127, 558.

(21) Chu, B. Laser Light Scattering; Academic Press: New York, 1991.

(22) Provencher, S. W. J. Chem. Phys. 1976, 64, 2772; Makromol. Chem. 1979, 180, 201

(23) Stockmayer, W. H.; Schmidt, M. Pure Appl. Chem. 1982, 54, 407; Macromolecules 1984, 17, 509.

(24) Vethamuthu, M. S.; Almgren, M.; Dubin, P. L.; Li, Y., unpublished.

(25) Li, Y.; Dubin, P. L.; Havel, H. A.; Edwards, S. L.; Dautzenberg, H. Langmuir, submitted.

(26) de Gennes, P.-G. Scaling Concepts in Polymer Physics; Cornell University Press: Ithaca, NY, 1979.

(27) Overbeek, J. Th. G.; Voorn, M. J. J. Cell. Comp. Physiol. 1957, 49, (Suppl. 1), 7 .

(28) Veis, A.; Aranyi, C. J. Phys. Chem. 1960, 64, 1203.

(29) Tainaka, K. J. Phys. Soc. Jpn. 1979, 46, 1899. 